\title{
INDICADORES DE PRODUÇÃO CIENTÍFICA - SÉRIE HISTÓRICA 2000-2004 CEFET-RN
}

\author{
José Yvan Pereira Leite ${ }^{1}$ \\ André Luís Calado de Araújo ${ }^{2}$ \\ Jerônimo Pereira dos Santos ${ }^{3}$ \\ Glauco Teixeira do Monte $^{4}$
}

\author{
${ }^{1}$ Prof. Área de Recursos Naturais e Mestre em Eng. Química - leite@cefetrn.br \\ ${ }^{2}$ Prof. da Área de Recursos Naturais e Doutor em Eng. Sanitária - acalado@cefetrn.br \\ ${ }^{3}$ Prof. da Área de Recursos Naturais e Mestre em Geociências - jeronimo@cefetrn.br \\ ${ }^{4}$ Assistente Administrativo e Especialista em Inteligência Competitiva - glauco@cefetrn.br
}

Recebido em agosto/2004 e Aceito em outubro/2004

\begin{abstract}
RESUMO
Este trabalho apresenta um resgate histórico da implantação das ações da pesquisa no CEFET-RN. Mostra a distribuição dos grupos de pesquisa em atuação em 2004 e analisa a distribuição por gerência educacional, por sexo, pela inserção docente em grupo de pesquisa e pela titulação. Estes dados são confrontados com a produtividade dos pesquisadores em relação à gerência educacional e ao grupo em que está vinculado. É observado um crescimento de $155 \%$ dos grupos de pesquisa, atualmente em número de 23 , como também um aumento significativo da titulação e a produtividade crescem suavemente. Cerca de $27 \%$ dos docentes estão inseridos nos grupos de pesquisa, sendo que a Gerência de Recursos Naturais apresenta 80\% dos seus docentes em grupos e sua produção intelectual representa $56 \%$ da produção Institucional no período de 2000 julho/2004. A relação de doutores e estudantes por grupo de pesquisa é de 0,6 com crescimento, respectivamente de $27,2 \%$ e $149,5 \%$ no período de $2002-$ julho/2004.
\end{abstract}

Palavras Chaves: Grupos de Pesquisa; pesquisador; produtividade; desenvolvimento; CEFET-RN.

\section{SCIENTIFIC PRODUCTION INDICATORS - HISTORICAL SERIE 2000-2004 IN CEFET-RN}

\begin{abstract}
This work presents a historical rescue of the implantation of research actions in CEFETRN. It shows the distribution of research groups in performance in 2004 and analyzes the distributions for educational management, sex, the teaching insertion in group of research and post-graduation titles. These data were collated from the productivity of the researchers in relation to the educational management and the group where it is tied. A growth of $155 \%$ of the groups of research was observed, reaching 23 groups, as also a significant increase of formation in post-graduation courses. Productivity grows softly. About $27 \%$ of the professors are inserted in research groups, and the Department of Natural Resources, for example, presents $80 \%$ of its professors in groups and its intellectual production represents $56 \%$ of the whole Institution in theperiod of 2000 julho/2004. The relation of doctors and students per group of research is 0.6 with growth, respectively of $27.2 \%$ and $149.5 \%$ in the period of 2002 -julho/2004.
\end{abstract}

Key-words: Research groups; researcher; research index; development; CEFET-RN. 


\section{INDICADORES DE PRODUÇÃO CIENTÍFICA - SÉRIE HISTÓRICA 2000-2004 CEFET-RN}

\section{INTRODUÇÃO}

Indicadores de produção científica têm sido amplamente divulgados como índices associados ao desenvolvimento de países, tendo em vista que a sociedade vive uma era do conhecimento.

As nações desenvolvidas e em desenvolvimento, tem aportado recursos para a formação de recursos humanos capacitados para os novos desafios, bem como para a pesquisa científica e aplicada.

Neste contexto, o Brasil tem realizado esforços no sentido de melhorar a qualificação de pesquisadores e aportar recursos para a pesquisa.

Este trabalho apresenta uma discussão da inserção da pesquisa realizada no Centro Federal de Educação Tecnológica do Rio Grande do Norte (CEFET-RN) no período de 2000 até julho de 2004 nos grupos de pesquisa cadastrados pela Instituição na plataforma Lattes do Conselho Nacional de Desenvolvimento Científico e Tecnológico (CNPq).

\section{UM BREVE HISTÓRICO}

A atividade de pesquisa do CEFET-RN foi iniciada formalmente a partir da publicação da revista ETFRN em 1985 na gestão da profa. Luzia Vieira de França, sendo seu editor a profa Maria Emília Guedes Gonçalves. A revista tinha como objetivo ser um veículo de comunicação da produção intelectual técnico-científica do público interno da Instituição (Revista da ETFRN, 1985).

A primeira publicação apresentou oito artigos, sendo um dos artigos do estudante Ricardo Luiz Vieira de França do curso de Eletrotécnica e a tabela 1 apresenta a lista dos trabalhos publicados.

Tabela 1 - Trabalhos publicados na Revista da ETFRN.

\begin{tabular}{|c|c|c|}
\hline Periódico & \multicolumn{2}{|r|}{ Autor } \\
\hline \multirow{8}{*}{$\begin{array}{c}\text { Revista da } \\
\text { ETFRN } \\
\text { (1985) }\end{array}$} & $\begin{array}{l}\text { Não se aprende nada inteiramente } \\
\text { novo }\end{array}$ & $\begin{array}{l}\text { Prof Marcondes } \\
\text { Guimarães }\end{array}$ \\
\hline & $\begin{array}{l}\text { A história do Brasil por uma visão } \\
\text { crítica }\end{array}$ & Prof Lailson de Almeida \\
\hline & $\begin{array}{l}\begin{array}{l}\text { Música, linguagem natural dos } \\
\text { sentimentos }\end{array} \\
\end{array}$ & Prof Pedro Ferreira \\
\hline & “Ufa! Que língua difícil”. & $\begin{array}{ll}\text { Profa Maria } & \text { Emília } \\
\text { Gonçalves }\end{array}$ \\
\hline & Organização escolar e democratização & $\begin{array}{l}\text { Prof Otávio Augusto de Araújo } \\
\text { Tavares }\end{array}$ \\
\hline & Descomplicação de tabu & Prof Walfredo Brasil \\
\hline & O Cooperativismo & Manoel Batista da Trindade \\
\hline & Fonte de alimentação estabilizada & Ricardo Luiz Vieira de França \\
\hline
\end{tabular}


Foi observado que os trabalhos publicados em 1985 não apresentaram artigos de cunho tecnológico, no entanto é observado um artigo de divulgação de matemática e um na área de eletrotécnica. O caráter individual dos trabalhos é evidenciado. Esses dados são bastante interessantes, visto que esta é uma Instituição marcada pelas suas ações vinculada à formação tecnológica.

A revista da ETFRN ficou no período de 1986-1989 sem publicar artigos e não foi identificado o motivo. A partir de 1990 ela volta a ser publicada e associada a esta ação, em 1992 é criada a coordenação de pesquisa, onde a tabela 2 apresenta aqueles que ocuparam este cargo até o ano de 2004.

Tabela 2 - Lista de coordenadores de pesquisa da Instituição no período de 19922004.

\begin{tabular}{|c|c|c|}
\hline Nome & Coordenação & Período \\
\hline $\begin{array}{l}\text { Zanoni Tadeu Saraiva dos } \\
\text { Santos }\end{array}$ & Pesquisa e Produção & $\begin{array}{l}08 / 1992- \\
04 / 1995\end{array}$ \\
\hline Dagmar Barbalho Azevedo & Pesquisa e Produção & $\begin{array}{l}04 / 1995- \\
04 / 1998\end{array}$ \\
\hline $\begin{array}{l}\text { Zanoni Tadeu Saraiva dos } \\
\text { Santos }\end{array}$ & Pesquisa e Produção & $\begin{array}{c}04 / 1998- \\
06 / 1998\end{array}$ \\
\hline $\begin{array}{l}\text { Renato Samuel Barbosa de } \\
\text { Araújo }\end{array}$ & Pesquisa e Desenvolvimento Tecnológico & $\begin{array}{l}06 / 1998- \\
03 / 2000\end{array}$ \\
\hline Régia Lúcia Lopes & Pesquisa e Desenvolvimento Tecnológico & $\begin{array}{l}03 / 2000- \\
09 / 2002\end{array}$ \\
\hline Sônia Cristina Ferreira Maia & Pesquisa e Desenvolvimento Tecnológico & $\begin{array}{c}09 / 2002- \\
03 / 2004\end{array}$ \\
\hline
\end{tabular}

Fonte - Gerência de Recursos Humanos e Direção Geral do CEFET-RN.

A inserção da pesquisa no organograma Institucional foi implantada na gestão do prof. Francisco das Chagas de Mariz Fernandes estando essa associada, na época, à Diretoria de Apoio e Extensão, dirigida pelo prof. Hudson Brandão. Em 2004 é criada a Diretoria de Pesquisa.

\section{RECURSOS HUMANOS E GRUPOS DE PESQUISA NO CEFET-RN}

Os primeiros grupos de pesquisa do CEFET-RN foram formalizados através do cadastro na plataforma Lattes em 2002, os quais totalizavam 9 (nove) e estava distribuída entre as áreas de engenharia, educação, ciência da terra e informática.... (Carta de Natal, 2002). Uma política de indução à formação de grupos de pesquisa realizada a partir de março de 2004 fez este número crescer para 23 grupos de pesquisas, distribuídos em todas Unidades Educacionais, os quais são mostrados na tabela 3.

A numeração apresentada na legenda associada aos grupos de pesquisa será usada ao longo deste trabalho.

O processo de indução da pesquisa praticado a partir de março de 2004 possibilitou um crescimento de 155\% em novos grupos de pesquisa na Instituição. Esta política está sustentada na associação entre a indução de editais de bolsa de pesquisador atrelada ao programa de iniciação científica, bem como a melhoria da qualidade do ensino 
possibilitado através de multiplicadores deste programa, levando a motivação dos alunos ao trabalho acadêmico.

Tabela 3 - Grupos de Pesquisa do CEFET-RN distribuídos nas Unidades Educacionais.

\begin{tabular}{|c|c|c|}
\hline UNIDADE & GRUPO DE PESQUISA & LEGENDA \\
\hline \multirow{6}{*}{$\begin{array}{l}\text { Gerência de } \\
\text { Formação } \\
\text { (GEFOR) }\end{array}$} & Núcleo de Pesquisa dm Educação & 1 \\
\hline & Caracterização de Solos Agrícolas & 2 \\
\hline & História dos Transportes Ferroviários & 3 \\
\hline & Cultura, Arte e Sociedade & 4 \\
\hline & Estudo da Transdisciplinaridade e da Complexidade & 5 \\
\hline & Núcleo de Pesquisa em Ensino e Linguagens & 6 \\
\hline \multirow{4}{*}{$\begin{array}{l}\text { Gerência de } \\
\text { Informática } \\
\text { (GEINF) }\end{array}$} & Núcleo de Desenvolvimento de Software - NUDES & 7 \\
\hline & Núcleo de Tecnologia Em Telemática - NUTEL & 8 \\
\hline & $\begin{array}{c}\text { Núcleo de Gestão e Tecnologias da Informação } \\
\text { Aplicadas aos Negócios }\end{array}$ & 9 \\
\hline & Núcleo de Desenvolvimento de Ambientes Virtuais & 10 \\
\hline \multirow{6}{*}{$\begin{array}{c}\text { Gerência de } \\
\text { Recursos Naturais } \\
\text { (GERN) }\end{array}$} & Análises de Águas, Efluentes e Estudos Costeiros & 11 \\
\hline & Grupo Interdisciplinar de Pesquisa Aplicada a Inclusão & 12 \\
\hline & Geoprocessamento & 13 \\
\hline & Processamento Mineral e Resíduos & 14 \\
\hline & Gestão Ambiental & 15 \\
\hline & Pesquisa Mineral & 16 \\
\hline \multirow{4}{*}{$\begin{array}{c}\text { Gerência de } \\
\text { Indústria (GETIN) }\end{array}$} & Núcleo de Pesquisa em Energia e Conforto Ambiental & 17 \\
\hline & Mecatrônica & 18 \\
\hline & $\begin{array}{l}\text { Núcleo de Pesquisas em Processos de Petróleo e Gás } \\
\text { Natural }\end{array}$ & 19 \\
\hline & Processamento de Materiais Metálicos e Não Metálicos & 20 \\
\hline $\begin{array}{c}\text { Gerência de } \\
\text { Serviços e Gestão } \\
\text { (GESEG) }\end{array}$ & Lazer e Gestão de Políticas Públicas e Privadas & 21 \\
\hline $\begin{array}{c}\text { Gerência de } \\
\text { Construção Civil } \\
\text { (GECON) }\end{array}$ & $\begin{array}{l}\text { Qualidade e Produtividade no Setor da Construção } \\
\text { Civil }\end{array}$ & 22 \\
\hline $\begin{array}{c}\text { Unidade } \\
\text { Descentralizada } \\
\text { (UNED) }\end{array}$ & $\begin{array}{c}\text { Núcleo de Estudos de Ciências e Tecnologias } \\
\text { Ambientais }\end{array}$ & 23 \\
\hline
\end{tabular}

O novo modelo pedagógico em discussão na Instituição objetiva apresentar à sociedade as novas diretrizes para as várias áreas em que atuam, sendo a pesquisa um desses elementos. A orientação visa à realização de pesquisa aplicada e voltada para a solução de problemas do plano local ao nacional. Com este enfoque, se entende que a sustentabilidade e a responsabilidade social deve governar as ações dos gestores para garantir nas suas ações o fomento de recursos para desenvolver um ensino de qualidade e a pesquisa pautada no desenvolvimento regional (Diretoria de Ensino - CEFET/RN, 2004). 


\section{GRUPOS DE PESQUISA NAS UNIDADES EDUCACIONAIS}

O fomento à pesquisa está associado à formação de grupos e a Instituição solicitou àqueles em atuação fossem cadastrados na base Lattes do Conselho Nacional de Desenvolvimento Científico e Tecnológico - CNPq. A figura 1 apresenta a distribuição dos grupos de pesquisa certificados pela Instituição, distribuídos nas Gerências Educacionais.

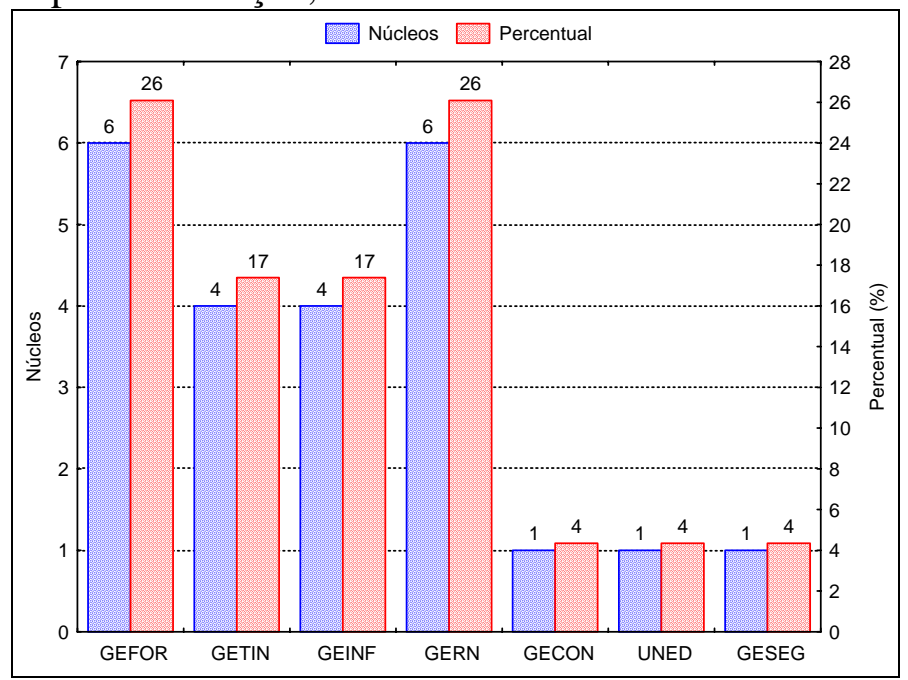

Figura 1 - Distribuição dos grupos de pesquisa por Gerências Educacionais (CEFET/RN). Fonte - Plataforma Lattes CNPq.

É observado na figura 1 que as Gerências de Construção Civil, de Serviços e a Unidade Descentralizada tem apenas 01 (um) grupo de pesquisa. As demais gerências têm entre 6 (seis) e 4 (quatro) grupos.

A distribuição por sexo, nos grupos de pesquisa e nas áreas educacionais, é mostrada na figura 2.

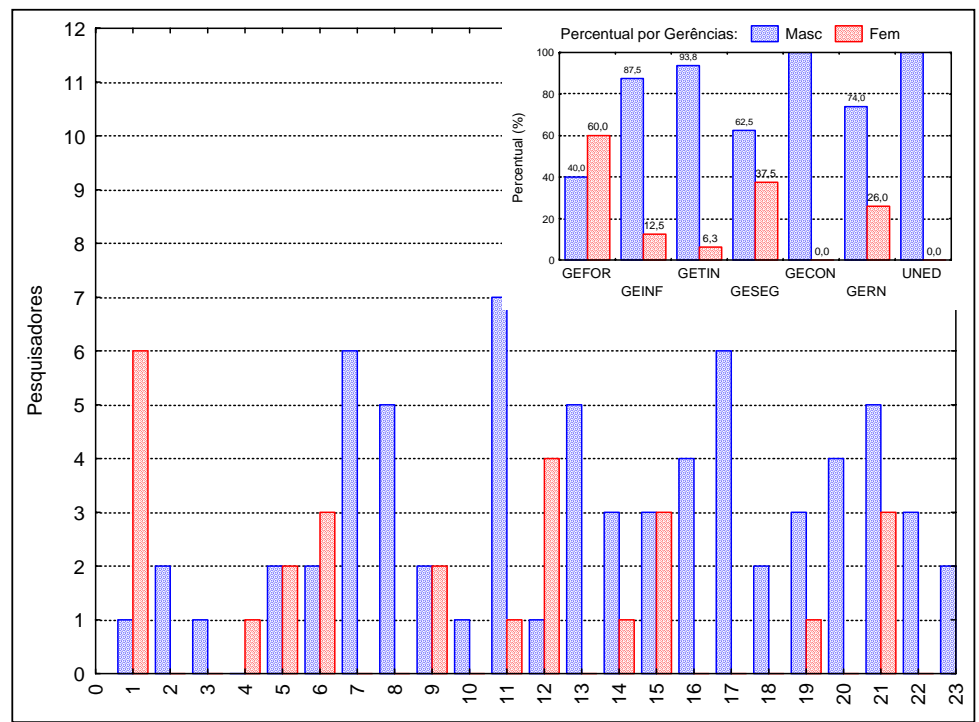

Figura 2 - Distribuição por sexo nos grupos de pesquisa e nas áreas educacionais no CEFET-RN.

A distribuição por sexo entre os grupos de pesquisa, nas gerências educacionais, mostra que apenas na GEFOR as mulheres são majoritárias, com $60 \%$ dos seus pesquisadores e 
nas outras gerências o predomínio masculino é determinante. A explicação para esta divisão esta vinculada a que ainda há um predomínio do sexo masculino nas áreas de ciências exatas e tecnológicas.

\section{TITULAÇÃO DOS PESQUISADORES EM GRUPOS DE PESQUISA}

A figura 3 mostra a titulação dos pesquisadores distribuídos pelas gerências.

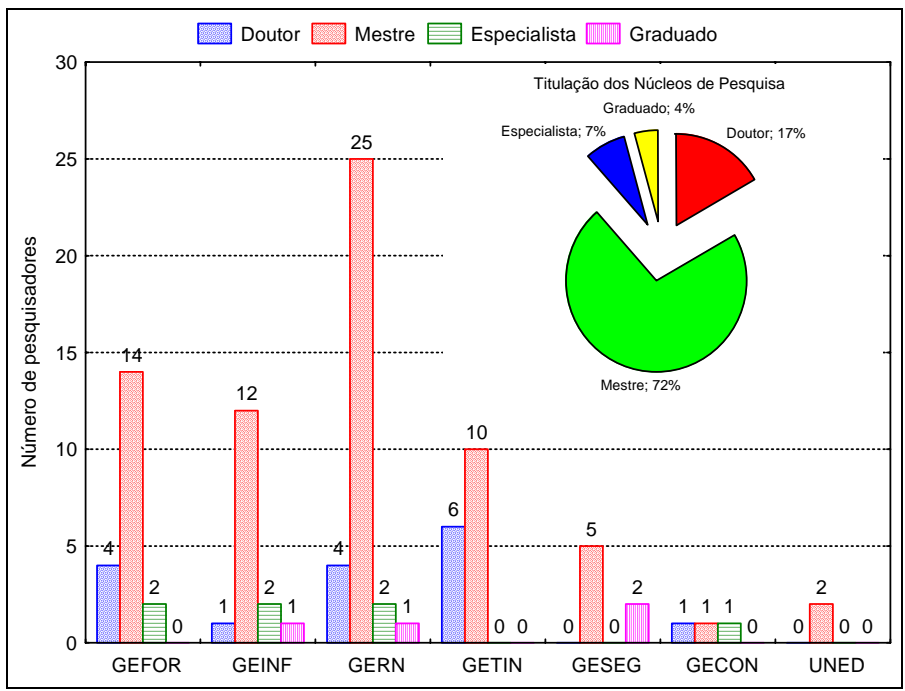

Figura 3 - Titulação dos pesquisadores nos grupos de pesquisa por Gerências Educacionais (CEFET/RN). Fonte - Plataforma Lattes CNPq.

Nota-se que $17 \%$ dos pesquisadores tem título de doutorado, $72 \%$ de mestrado e que $11 \%$ ainda estão como especialistas e graduados. É importante ressaltar que existe um número importante de pesquisadores em processo de capacitação, tendo em vista a obtenção de título de doutorado e mestrado. É observado que a área com melhor titulação atualmente é a GETIN.

A tabela 3 mostra a distribuição dos docentes nos grupos de pesquisa em relação a sua gerência educacional.

Tabela 3 - Distribuição de pesquisadores inseridos nas unidades do CEFET-RN. Fonte - GDRH e CNPq.

\begin{tabular}{|c|c|c|c|}
\hline Unidade & Docentes & Grupo & $\begin{array}{c}\% \\
\text { Distribuição }\end{array}$ \\
\hline SEDE & 288 & 93 & 32,29 \\
\hline UNED & 68 & 2 & 2,94 \\
\hline GEFOR & 113 & 20 & 17,70 \\
\hline GERN & 40 & 32 & 80,00 \\
\hline GECON & 30 & 3 & 10,00 \\
\hline GETIN & 37 & 16 & 43,24 \\
\hline GESEG & 34 & 7 & 20,59 \\
\hline GEINF & 33 & 16 & 48,48 \\
\hline
\end{tabular}


A Unidade Sede tem 32,29\% dos seus docentes incorporados em grupos de pesquisa, enquanto na UNED tem se 2,94\%. É importante ressaltar que na UNED a pesquisa foi induzida em março deste ano, tendo grupos em formação e docentes a serem inseridos nos respectivos grupos.

Na Universidade de São Paulo (USP) praticamente todos os professores participam de pelo menos 01 grupo de pesquisa (Folha de São Paulo, 2004), mostrando a necessidade de uma maior inserção dos professores do CEFET-RN em grupos de pesquisa.

O redimensionamento do projeto político-pedagógico do CEFET-RN (DE-CEFET-RN, 2004) coloca com clareza que a função docente deve contemplar de forma indissociável a unidade ensino-pesquisa. O contexto em questão mostra que as diretrizes do projeto pedagógico estão em processo de consolidação nas unidades da GERN (80\%), GEINF (48,48\%) e da GETIN (43,24\%), sendo necessário um trabalho maior nas unidades da UNED (2,94\%), da GECON (10,00\%), da GEFOR (17,70\%) e da GESEG (20,59\%), visando à ampliação e inserção do trabalho docente para a consolidação de grupos de pesquisas e que estes contribuam para o desenvolvimento do país.

\section{PRODUÇÃO E PRODUTIVIDADE EM PESQUISA}

A figura 4 apresenta a produção intelectual obtida através do currículo Lattes do CNPq com acesso no início de agosto de 2004.

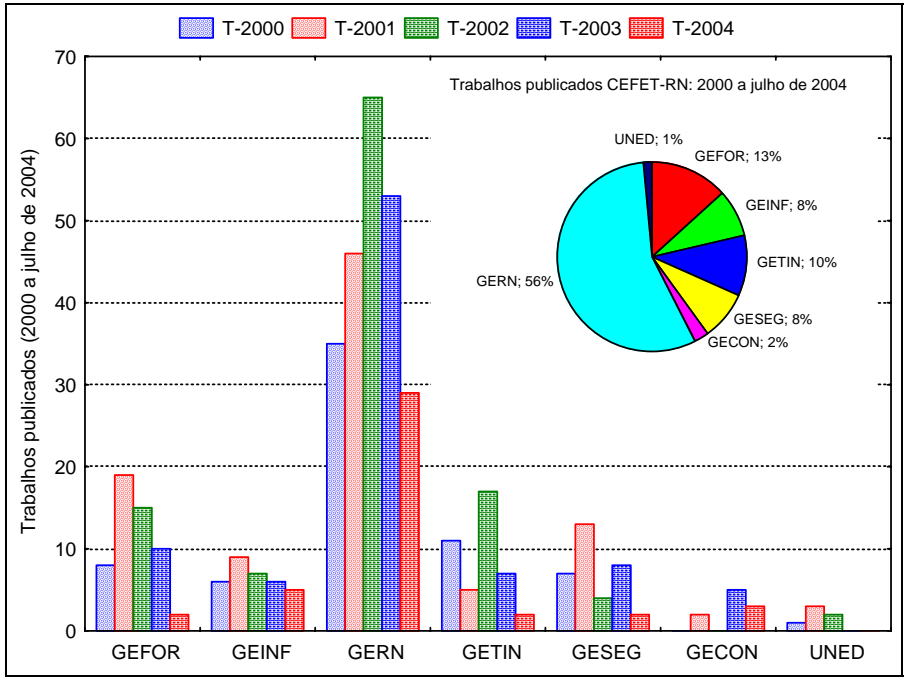

Figura 4 - Número de trabalhos publicados pelos pesquisadores nos grupos de pesquisa e sua distribuição percentual nas Gerências Educacionais (CEFET/RN). Fonte - Plataforma Lattes CNPq.

A figura 4 mostra que a Gerência de Recursos Naturais foi responsável por $56 \%$ da produção intelectual dos grupos de pesquisa do CEFET-RN no período em estudo (200007/2004), seguida pelas Gerências de Formação (13\%), de Indústria (10\%), de Informática (10\%), de Serviços e Gestão (8\%) e a Unidade Descentralizada (2\%). Analisando a distribuição da produção se observa que os grupos de pesquisas vinculados a GERN e GESEG apresentaram uma produção intelectual bem superior às outras gerências, tendo em vista o número de pesquisadores e a sua produtividade. 
Com o objetivo de avaliar a distribuição da produção intelectual dos pesquisadores nos grupos e seu reflexo nas unidades educacionais, foi efetuado no período (2000-julho/2004) consulta a plataforma Lattes verificando as publicações dos pesquisadores nas formas de trabalho completos e em resumo, sem avaliar o seu nível de impacto, tendo em vista que o objetivo deste trabalho é apresentar à comunidade o nível da inserção do CEFET-RN em pesquisa e desenvolvimento. A figura 5 apresenta a produtividade docente distribuída pelos grupos e unidades educacionais.

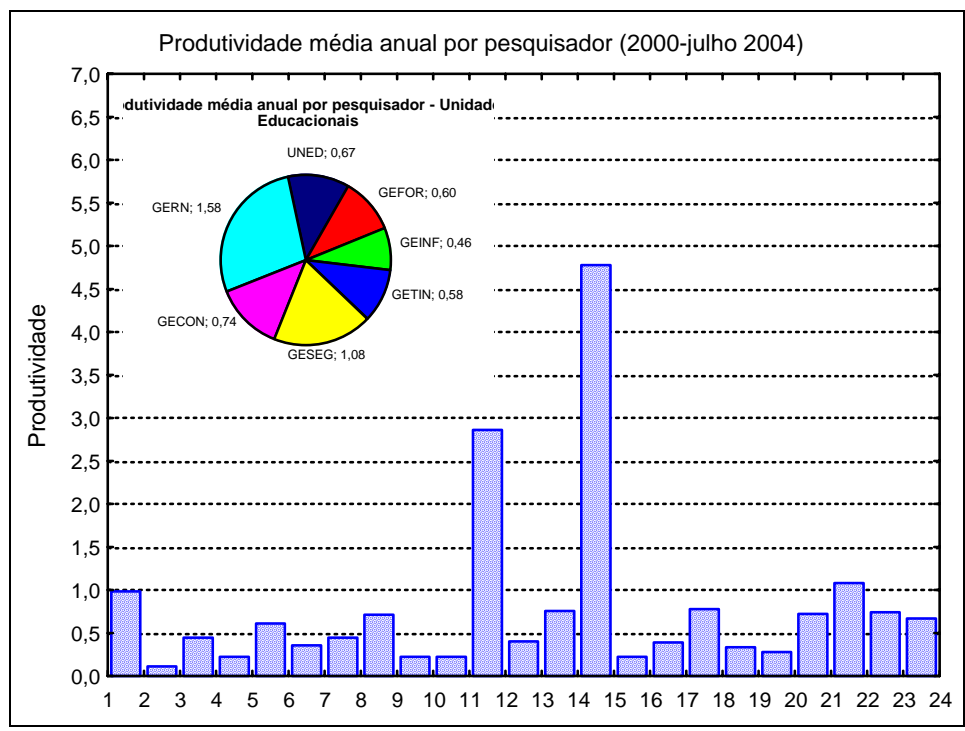

Figura 5 - Produtividade dos pesquisadores inseridos nos grupos de pesquisa e nas unidades educacionais. Legenda - Disponível na tabela 3 deste trabalho.

É importante ressaltar que a produtividade analisada contabiliza todos os trabalhos publicados, seja na forma de resumos, publicações em eventos e em periódicos, sendo esta classe com número bastante incipiente.

Um parâmetro interessante a analisar no Brasil, considerando a publicação de artigos em periódicos, mostra que na USP a produtividade média é de 0,4 enquanto os Institutos de Física (USP-São Carlos) e o Instituto de Química estes índices são de 3,9 e 3,4 respectivamente (Folha de São Paulo, 2004). No período avaliado um número reduzido de professores do CEFET-RN publicou em periódicos indexados, devendo ser objeto de suas políticas o estimulo a produção de artigos em periódicos indexados.

As médias de produtividade (trabalhos/ano) apresentadas no CEFET-RN estão oscilando em torno de 0,1 a 4,7 trabalhos por pesquisador/ano. Esta relação de produtividade deverá ser melhorada, tendo em vista que a análise foi efetuada até julho/2004, devido às ações de indução à pesquisa que vêm sendo desenvolvidas.

No Brasil vem sendo realizado um grande esforço para a consolidação da pesquisa brasileira que no período entre 1997-2001 aparece com 0,5\% da produção intelectual do mundo, tendo publicado 188 artigos em periódicos indexados (L. Marques, 2004). A consolidação do trabalho de pesquisa em desenvolvimento na Instituição deve iniciar esforços para publicar seus trabalhos em periódicos indexados, tendo em vista contribuir com os índices internacionais onde estes têm impactado positivamente no desenvolvimento regional. 
A tabela 4 mostra a evolução do último senso realizado pelo CNPq nos grupos de pesquisa do CEFET-RN.

Tabela 4 - Indicadores de produtividade no período dos censos de 2002 e o de julho/2004.

\begin{tabular}{c|c|c|c|c|c|c|c|c|c|c|c}
\hline & $(\mathbf{G})$ & $(\mathbf{P})$ & $(\mathbf{D})$ & $(\mathbf{E})$ & $(\mathbf{T})$ & $(\mathbf{L})$ & $\mathbf{P} / \mathbf{G}$ & $\mathbf{D} / \mathbf{G}$ & $\mathbf{E} / \mathbf{G}$ & $\mathbf{T} / \mathbf{G}$ & $\mathbf{L} / \mathbf{G}$ \\
\hline 2002 & 9 & 35 & 4 & 8 & 1 & 14 & 3,9 & 0,4 & 0,9 & 0,1 & 1,6 \\
\hline $07 / 2004$ & 23 & 85 & 13 & 51 & 3 & 54 & 3,7 & 0,6 & 2,2 & 0,1 & 2,3 \\
\hline $\begin{array}{c}\text { Variação } \\
\text { (\%) }\end{array}$ & 155,6 & 142,9 & 225 & 537,5 & 200 & 385,7 & 5,0 & 27,2 & 149,5 & 17,4 & 50,9 \\
\hline
\end{tabular}

Legenda - G (Grupos); P (Pesquisadores); D (Doutor); E (Estudante); T (Técnico); Linhas de Pesquisa (L).

A tabela 4 mostra uma variação percentual positiva na criação de novos grupos de pesquisa na ordem de $155 \%$ e de pesquisadores em $143 \%$, bem como na melhor qualificação dos seus pesquisadores com um aumento de 225\% dos doutores. Outro dado bastante importante é observado no crescimento de 537,5\% na participação de estudantes, refletindo em um maior número de linhas de pesquisa da ordem de 385,7\%.

A relação de pesquisadores por grupo teve variação de 5\%, tendo 3,7 pesquisadores/grupo. No que se refere à relação de doutores por grupo a relação cresceu em $27,2 \%$, mas ainda é necessário realizar esforços para esta relação ficar próxima ao índice dos pesquisadores/grupo. Da mesma forma, a relação dos estudantes por grupos precisa ter um valor de pelo menos um pesquisador para cada estudante, assim essa relação deve ser objeto de incentivo à introdução de mais estudantes nos grupos de pesquisa. Esta política se associa à melhoria da qualidade do ensino.

Como existe uma política de indução a pesquisa estes números são melhores do que estão publicados, tendo em vista que os pesquisadores não adquiriram o hábito de inserir sua produção acadêmica e os alunos que orientam no currículo Lattes. Assim, é identificado que existe necessidade de realizar solicitações permanentes para corrigir esses dados, os quais melhor refletirão a qualidade da pesquisa realizada no CEFET-RN.

Observando a inserção de técnicos nos grupos, esta é ainda bastante baixa, precisando o governo abrir mais postos de trabalhos nestas áreas, as quais influenciarão no ensino realizado na Instituição e serão determinantes no desenvolvimento do trabalho científico e de inovação tecnológica.

Na Instituição há esforços para realizar a associação do programa de pesquisa com as políticas de empreendedorismo, através da vinculação do Núcleo de Incubação Tecnológica (NIT) à Diretoria de Pesquisa, a qual vem aproximando as ações do desenvolvimento acadêmico com a necessidade da consolidação de produtos, pois os projetos de pesquisas bem sucedidos devem ser objeto da criação de empreendimentos incubados, os quais deverão ser alternativas de desenvolvimento sustentável para a formação de novos talentos e é claro, da região em que está inserido o CEFET-RN.

\section{POLÍTICAS CIENTÍFICAS PARA A REDE - CEFET}

A cooperação científica entre os CEFETs objetivando o sistemático das ações de pesquisa e inovação deve esta associado a criação de fóruns comuns, quais devem ser em evento técnico científico anual para propiciar a integração dos seus pesquisadores (docentes, 
administrativos e estudantes), conhecimento do potencial instrumental, das linhas de pesquisas afins, de buscas alternativas para as soluções conjuntas dos problemas regionais, através de participações em projetos de pesquisa estimulados pelos arranjos produtivos, em editais dos fundos públicos de apóio a pesquisa e de fontes alternativas do terceiro setor para o financiamento de inovações tecnológicas.

O processo de aprendizagem do trabalho a distância deve ser consolidado, visto que esta prática aumenta a participação nos eventos organizados em conjunto e que todas as Instituições objeto desta discussão tem disponíveis estações de vídeo-conferência. É preciso realizar processos participativos educativos para otimizar os recursos e melhor utilizá-los.

O periódico, de preferência em área de pesquisa consolidada, deve ser viabilizado com conselho editorial representativo dos grupos de pesquisa com produtividade acadêmica medida através da base Lattes do CNPq. Este periódico deve difundir os conhecimentos científicos e de inovação desenvolvidos majoritariamente nos CEFETs.

Estas Instituições passam por um novo momento histórico, seja pelo re-ordenamento da política pedagógica pelas exigências da formação de profissionais em terceiro nível, sua qualidade estão vinculadas à inserção dos seus quadros a pesquisa e inovação com foco no desenvolvimento de produtos para a solução de demandas da sociedade brasileira.

O programa das Nações Unidas para o desenvolvimento publicou em seu site um ranking dos indicadores de desenvolvimento humano, onde o Brasil se coloca em $65^{\circ}$ colocação. A tabela 5 mostra os indicadores na área tecnológica (Human Devolopment Report - ONU, 2004).

Tabela 5 - Indicadores de Desenvolvimento Humano. Fonte - Programa de Desenvolvimento Humano das Nações Unidas.

\begin{tabular}{|c|c|c|c|c|c|c|c|c|c|}
\hline $\begin{array}{c}\text { IDH } \\
\text { (Posição) }\end{array}$ & \multicolumn{2}{|c|}{$\begin{array}{c}\text { Telefones } \\
(1.000 \text { hab.) }\end{array}$} & \multicolumn{2}{|c|}{$\begin{array}{c}\text { Celular } \\
(1.000 \text { hab.) }\end{array}$} & \multicolumn{2}{c|}{$\begin{array}{c}\text { Internet } \\
(1.000 \text { hab.) }\end{array}$} & $\begin{array}{c}\text { Patentes } \\
\text { (milh. } \\
\text { hab.) }\end{array}$ & $\begin{array}{c}\text { P\&D } \\
\text { (\% GDP) }\end{array}$ & $\begin{array}{c}\text { Cientistas e Eng. } \\
\text { em P\&D (milh. } \\
\text { hab.) }\end{array}$ \\
\cline { 2 - 10 } & 1990 & 2000 & 1990 & 2000 & 1990 & 2000 & 1999 & $1996-2000$ & $1996-2000^{\text {a }}$ \\
\hline 65 & 65 & 218 & - & 167 & - & 46,6 & 3 & 0,8 & 323 \\
\hline
\end{tabular}

Legenda - IDH (índice de Desenvolvimento Humano);

Os índices apresentados mostram evoluções excepcionais no Brasil ao acesso aos sistemas de informações, no entanto estes não refletem a inserção para a geração de patentes e de investimentos em Pesquisa \& Desenvolvimento. Os CEFETs tem responsabilidade direta na inversão destes índices, tendo em vista que dentre os seus objetivos esta a motivação para uma educação de qualidade e o desenvolvimento tecnológico.

A Declaração do Milênio foi aprovada pelas Nações Unidas em 2000, sendo o Brasil um dos signatários, esta são um conjunto de 8 (oito) macro-objetivos a serem atingidos até 2015, a saber: 1- Erradicar a extrema pobreza e a fome; 2- Atingir o ensino básico universal; 3- Promover a igualdade de gênero e a autonomia das mulheres; 4- Reduzir a mortalidade infantil; 5- Melhorar a saúde materna; 6- Combater o HIV/Aids, a malária e outras doenças; 7- Garantir a sustentabilidade ambiental; 8- Estabelecer uma Parceria Mundial para o Desenvolvimento (Declaração do Milênio das Nações Unidas, 2000 Objetivos de Desenvolvimento do Milênio). 
O item 8 (oito) deve ser trabalhado de forma cooperada na RedeNet (Rede Nordeste dos CEFETs), objetivando contribuir com as políticas acordadas pelo país e estabelecer, de forma coordenada ações que minorem as diferenças entre os cidadãos brasileiros, através da capacitação para o desenvolvimento.

\section{CONSIDERAÇÕES FINAIS}

Este trabalho apresentou um breve histórico da pesquisa no CEFET-RN e a situação dos grupos de pesquisa em atividade até julho de 2004, bem como mostra que a Instituição vem melhorando seus índices na formação de grupos de pesquisa (23 grupos e crescimento de 155\%), de produtividade (cerca de 1 trabalho/pesquisador ano), da relação pesquisador por aluno é de 0,6 e que deve está próxima de 1 (um), tendo em vista que os pesquisadores ainda não têm hábito da atualização permanente do currículo na base Lattes do CNPq.

Os dados disponibilizados neste trabalho devem ser utilizados pelos pesquisadores e dirigentes, tendo em vista realizar um planejamento adequado a atender as suas necessidades, bem como apresentar a sociedade o potencial que a Instituição tem, para realizar as demandas da sociedade visando cooperar para um ambiente propicia ao desenvolvimento sustentável.

\section{REFERÊNCIAS BIBLIOGRÁFICAS}

Carta de Natal - Documento de Política de Pesquisa do CEFET-RN, Natal, abril/2002, pp. 1-22.

Moura, D. H., Redimensionamento do Projeto Político-pedagógico do CEFET-RN, $2^{\text {a }}$ Bloco Temático - O papel da Educação Profissional e a função do CEFET-RN na sociedade: possibilidades e limitações. Diretoria de Ensino - CEFET/RN,. Natal, 2004. pp 30.

Human Devolopment Report - $\quad$ United Nations. http://www.undp.org/hdr2003/indicator/indic_108_1_1.html. Acesso em agosto de 2004.

Marques, L., Indicadores - Uma Prova de Qualidade. In: Revista Pesquisa FAPESP. N ${ }^{0}$ 12, agosto 2004. pp. 24-27.

Objetivos de Desenvolvimento do Milênio das Nações Unidas http://www.pnud.org.br/odm/index.php?lay=odmi\&id=odmi. Acesso em agosto de 2004.

Plataforma Lattes - CNPq. http: //lattes.cnpq.br. Acesso em agosto de 2004.

Revista da ETFRN, N ${ }^{0}$ 1, agosto/1985. pp. 1-30.

USP 70 Anos, Folha de São Paulo - Especial, 23 de janeiro de 2004.

\section{AGRADECIMENTOS}

Os autores expressam seus agradecimentos aos colegas servidores do CEFET-RN, Auridan Dantas de Araújo e Maria Auxiliadora Pereira de Lira, pelo acesso aos dados Institucionais, bem como às bolsistas do CNPq, Ana Cláudia Fernandes da Silva e Letícia Rosana Silva do Nascimento, pela contribuição na coleta dos dados deste trabalho. 